\title{
Antioxidant enzymes induced by repeated intake of excess energy in the form of high-fat, high-carbohydrate meals are not sufficient to block oxidative stress in healthy lean individuals
}

\author{
Sangbin Lim $^{1}$, Hyeran Won ${ }^{1}$, Yeonghwan $\mathrm{Kim}^{1}$, Miran $\mathrm{Jang}^{1}$, K. R. Jyothi ${ }^{1}$, Youngseol Kim ${ }^{3}$, \\ Paresh Dandona ${ }^{1,2}$, Joohun $\mathrm{Ha}^{1}$ and Sung Soo Kim ${ }^{1 *}$ \\ ${ }^{1}$ Department of Biochemistry and Molecular Biology (BK21 Project), Medical Research Center for Bioreaction to Reactive \\ Oxygen Species and Biomedical Science Institute, School of Medicine, Kyung Hee University, No. 1 Hoegi-dong, \\ Dongdaemun-gu, Seoul 130-701, South Korea \\ ${ }^{2}$ Division of Endocrinology, Diabetes and Metabolism, State University of New York at Buffalo and Kaleida Health, \\ 3 Gates Circle, Buffalo, NY 14209, USA \\ ${ }^{3}$ Department of Endocrinology and Metabolism, School of Medicine, Kyung Hee University, No. 1 Hoegi-dong, \\ Dongdaemun-gu, Seoul 130-701, South Korea
}

(Received 12 October 2010 - Revised 14 March 2011 - Accepted 14 March 2011 - First published online 8 June 2011)

\begin{abstract}
It has been reported that high-fat, high-carbohydrate (HFHC) meals increase oxidative stress and inflammation. We examined whether repeated intake of excess energy in the form of HFHC meals alters reactive oxygen species (ROS) generation and the expression levels of antioxidant enzymes and mitochondrial proteins in mononuclear cells, and to determine whether this is associated with insulin resistance. We recruited healthy lean individuals $(n$ 10). The individuals were divided into two groups: one group $(n 5)$ ingested $10878 \cdot 4 \mathrm{~kJ} / \mathrm{d}$ (2600 kcal/d; 55-70\% carbohydrate, 9.5-16\% fat, 7-20\% protein) recommended by the Dietary Reference Intake for Koreans for $4 \mathrm{~d}$ and the other group ( $n$ 5) ingested a HFHC meal containing $14644 \mathrm{~kJ} / \mathrm{d}(3500 \mathrm{kcal} / \mathrm{d})$. Then, measurements of blood insulin and glucose levels, together with suppressor of cytokine signalling-3 (SOCS-3) expression levels, were performed in both groups. Also, cellular and mitochondrial ROS levels as well as malondialdehyde (MDA) levels were measured. Expression levels of cytosolic and mitochondrial antioxidant enzymes, and mitochondrial complex proteins were analysed. Repeated intake of HFHC meals induced an increase in homeostasis model of assessment-insulin resistance (HOMA-IR), together with an increase in SOCS-3 expression levels. While a single intake of the HFHC meal increased cytosolic and mitochondrial ROS, repeated intake of HFHC meals reduced them and increased the levels of MDA, cytosolic and mitochondrial antioxidant enzymes, and several mitochondrial complex proteins. Repeated intake of HFHC meals induced cellular antioxidant mechanisms, which in turn increased lipid peroxidation (MDA) and SOCS-3 expression levels, induced hyperinsulinaemia and increased HOMA-IR, an index of insulin resistance. In conclusion, excess energy added to a diet can generate detrimental effects in a short period.
\end{abstract}

Key words: Reactive oxygen species: Antioxidant enzymes: High-fat, high-carbohydrate meals: Mitochondrial electron transport chain

It is well known that high-fat, high-carbohydrate (HFHC) meals increase oxidative stress and inflammation ${ }^{(1-3)}$. Such meals also increase plasma concentrations of endotoxin (lipopolysaccharide), the expression of its receptor toll-like receptor (TLR)-4 and the expression of suppressor of cytokine signalling-3 (SOCS-3) that interferes with insulin signal transduction and may mediate insulin resistance ${ }^{(4)}$. Consistent with these observations, experimental studies on the effects of HFHC meals in animals and human subjects have shown that such meals are associated with insulin resistance ${ }^{(5-8)}$. For instance, acute bouts of HFHC meals over a period of $5 \mathrm{~d}$ are associated with the development of significant insulin resistance in healthy lean individuals ${ }^{(9,10)}$. The mechanisms underlying the induction of insulin resistance are not understood. Oxidative

Abbreviations: COX, cyclo-oxygenase subunit; HFHC, high fat, high carbohydrate; HOMA-IR, homeostasis model of assessment-insulin resistance; KDRI, Dietary Reference Intake for Koreans; mETC, mitochondrial electron transport chain; MNC, mononuclear cells; ND1, NADH dehydrogenase 1; NDUFB, NADH dehydrogenase [ubiquinone] $1 \beta$ sub-complex subunit; ONOO ${ }^{-}$, peroxynitrite; Prx, peroxiredoxin; Rieske Fe-S, cytochrome $b-c 1$ complex subunit; ROS, reactive oxygen species; SDHB, succinate dehydrogenase complex subunit B; SOCS-3, suppressor of cytokine signalling-3; SOD, superoxide dismutase; TBA, thiobarbituric acid; TFAM, mitochondrial transcription factor A.

*Corresponding author: S. S. Kim, fax +82 29598168 , email sgskim@khu.ac.kr 
stress and inflammation may play a role since SOCS-3 is induced by pro-inflammatory cytokines.

Many studies have demonstrated the generalised mitochondrial dysfunction in type 2 diabetic patients ${ }^{(11)}$. Because over-nutrition leads to insulin resistance, it is possible that HFHC meals can induce mitochondrial dysfunction. Overfeeding can also cause an increase in the expression of mitochondrial electron transport chain (mETC) complexes ${ }^{(12,13)}$. This may be relevant to postprandial reactive oxygen species (ROS) generation and oxidative stress. Oxidative stress results when the generation of ROS exceeds the capacity of the cellular antioxidant defence system to eliminate ROS. The activity of the antioxidant defence system is particularly relevant to increased ROS generation at the mitochondrial and cellular levels ${ }^{(14,15)}$.

Although there have been many reports suggesting that macronutrient intake induces oxidative stress and insulin resistance, there are no data related to the response of antioxidant defence mechanisms to oxidative stress during macronutrient intake. We hypothesised that repeated intake of excess energy in the form of HFHC meals would increase oxidative stress and insulin resistance, and alter the expression of antioxidant enzymes and mETC complex subunits.

\section{Methods}

\section{Ethics}

The present study was conducted according to the guidelines laid down in the Declaration of Helsinki and all procedures involving human individuals were approved by the Institutional Review Board and the Ethical Committee of Kyung Hee University Hospital. Before recruitment and enrolment into the study, the purpose of the study was explained to each prospective candidate in the local language, and a written informed consent was obtained from all individuals.

The trial is registered as ISRCTN04309078 (www.controlledtrials.com).

\section{Individual recruitment}

Individuals were randomised. Individuals were eligible if they were healthy volunteers, aged 28-32 years and BMI ranged from 18.5 to $22.9 \mathrm{~kg} / \mathrm{m}^{2}$. However, individuals were not eligible if their complete blood count was more than $6000 / \mu \mathrm{l}$ (leucocytes), their fasting blood sugar was more than $1000 \mathrm{mg} / 1$, or their HbA1c was more than $6.0 \%$, and if they have some metabolic disease or family history of diabetes. Finally, ten healthy, lean male Korean individuals were recruited in the present study. There were no significant differences in weight, BMI and blood pressure of individuals. In the present study, female volunteers could not be included because they have oestrogen that may have an effect on glucose and insulin metabolism. However, we will include female subjects in future studies since they can also be affected by the intake of excess energy in the form of a HFHC meal.

\section{Diet design}

A total of ten healthy, lean male Korean individuals, tested at the Kyung Hee University Hospital, were enrolled in the present study. The Dietary Reference Intake for Koreans (KDRI) guidelines recommends $10878.4 \mathrm{~kJ} / \mathrm{d}(2600 \mathrm{kcal} / \mathrm{d} ; 55-70 \%$ carbohydrate, $9 \cdot 5-16 \%$ fat and $7-20 \%$ protein) for Korean male individuals ${ }^{(16)}$. Individuals started a standardised diet for $7 \mathrm{~d}$ with control meals for breakfast, lunch and dinner, based on the KDRI guidelines. Blood samples were collected at $2 \mathrm{~h}$ after lunch on days 1 and 7 during the intake period of KDRI control meals for the standardised diet. The KDRI control meal contained 110.67 (SD 11.55) g carbohydrates, 20.6 (SD 2.42) g fat, and 36.63 (SD 6.34) g protein, with an energy content between $3556 \cdot 4 \mathrm{~kJ}(850 \mathrm{kcal})$ and $3765.6 \mathrm{~kJ}$ (900 kcal). After the standardised diet, five individuals continually ingested KDRI control meals for breakfast, lunch and dinner, and the other five individuals ate HFHC meals only for lunch daily for $4 \mathrm{~d}$. This meal contained $7531.2 \mathrm{~kJ}$ (1800 kcal) (Burger King Double Whopper burger, regular French fries, a small coke and regular onion rings; this meal contains $173 \mathrm{~g}$ carbohydrates, $93 \mathrm{~g}$ fat and $65 \mathrm{~g}$ protein). Therefore, the KDRI control group ingested approximately $10878.4 \mathrm{~kJ} / \mathrm{d}(2600 \mathrm{kcal} / \mathrm{d} ; 340 \mathrm{~g}$ carbohydrate, $66 \mathrm{~g}$ fat and $120 \mathrm{~g}$ protein), and the other group ingested approximately $14644 \mathrm{~kJ} / \mathrm{d}(3500 \mathrm{kcal} / \mathrm{d} ; 410 \mathrm{~g}$ carbohydrate, $130 \mathrm{~g}$ fat and $130 \mathrm{~g}$ protein) for $4 \mathrm{~d}$. Blood samples were collected at $2 \mathrm{~h}$ after lunch on days 9 and 11 during the intake period of KDRI meals or HFHC meals (Fig. 1(a)).

To supply the balanced nutrition, we prohibited individuals from any meals and drinks that we did not serve during the study. In addition, to prohibit unnecessary action, all individuals stayed in a camp during the study.

\section{Randomisation}

The study was a randomised controlled trial with two arms wherein we evaluated the effect of repeated HFHC meals in comparison with control meals. A randomisation list on the basis of BMI was generated using an in-house computerised program, and there was no significant difference in BMI between the two groups. All individuals were listed randomly using letter code. These processes were conducted by an administrator. Personal information was not publicised to investigators or anyone.

\section{Oral glucose tolerance test}

Oral glucose tolerance test was performed on the mornings of days 8 and 12. Individuals were examined after an overnight fasting at Kyung Hee University Medical Center and had an indwelling intravenous cannula inserted into their anterior cubital vein. They then ingested $75 \mathrm{~g}$ of glucose. Individuals rested in a semi-recumbent position until the end of the test, and blood samples for glucose measurements were collected at 30, 60, 90, and $120 \mathrm{~min}$. Each individual was subjected to a glucose challenge before and after the intake of HFHC or KDRI meals for lunch for four consecutive days. Glucose 
(a)
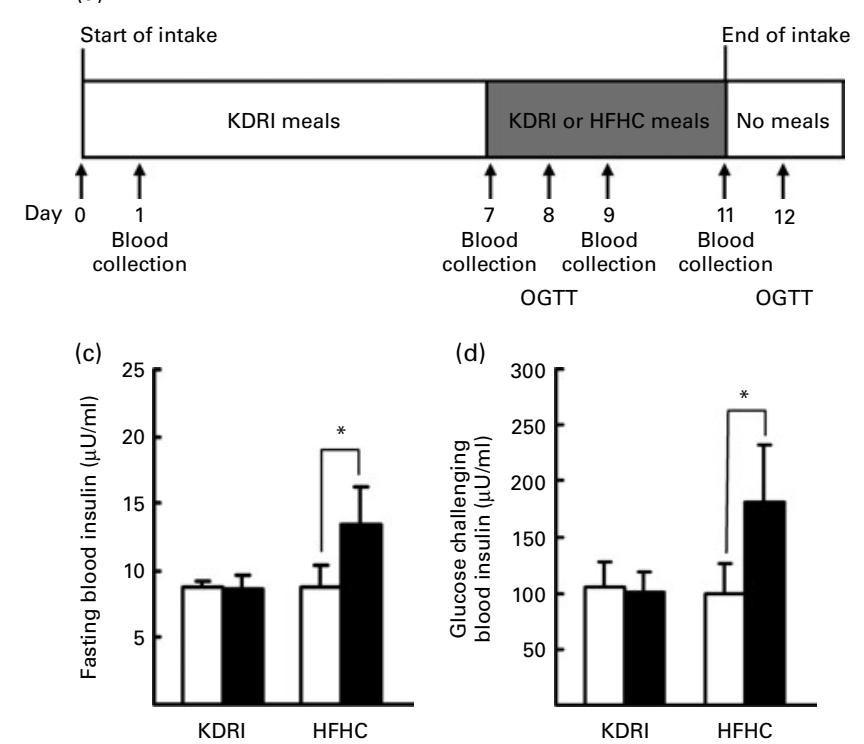

(d)

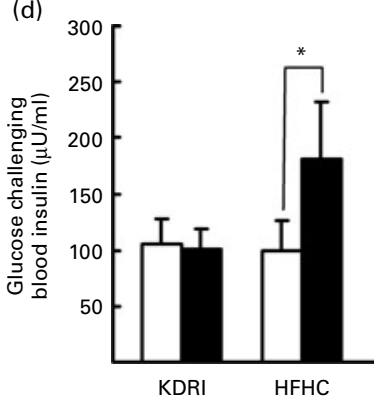

(b)

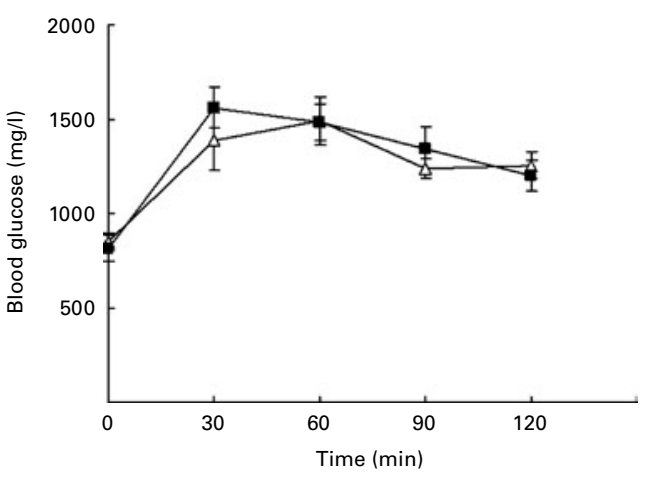

(e)

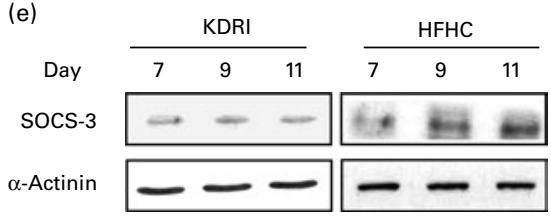

Fig. 1. Hyperinsulinaemia and insulin resistance after repeated intake of high-fat, high-carbohydrate (HFHC) meals. (a) Schematic diagram of the experimental design. Blood samples were collected at $2 \mathrm{~h}$ after lunch on blood collection days. Oral glucose tolerance test (OGTT) was performed at morning on each of the days. (b) OGTT glucose concentrations measured on days 8 ( $\square$ ) and 12 (घ) were expressed; - $\triangle-$, Dietary Reference Intake for Koreans (KDRI); - $\square-$, HFHC. Blood insulin levels were measured following (c) fasting and (d) glucose challenge. (e) Western blot analysis. The protein expression level of suppressor of cytokine signalling-3 (SOCS-3) following HFHC meals was compared with that following KDRI meals. $\alpha$-Actinin was used as the loading control. *Mean values were significantly different from those of $\mathrm{KDRI}$ meals $(P<0.05) .1 \mu \mathrm{U} / \mathrm{ml}$ insulin $=6.945 \mathrm{pmol} / \mathrm{l}$.

was measured at each time point while insulin was measured at 0 and $30 \mathrm{~min}$.

\section{Mononuclear cell isolation}

Blood samples were collected in tubes containing Na-EDTA as an anticoagulant; $3.5 \mathrm{ml}$ of the anticoagulated blood sample was carefully layered over $3.5 \mathrm{ml}$ of polymorphonuclear leucocyte isolation medium (Cedarlane Laboratories, Hornby, ON, Canada). Samples were centrifuged at $450 \mathrm{~g}$ in a swing-out rotor for $30 \mathrm{~min}$ at $22^{\circ} \mathrm{C}$. At the end of centrifugation, two bands were separated out at the top of the erythrocyte pellet. The top band consisted of mononuclear cells (MNC), whereas the bottom band consisted of polymorphonuclear leucocytes. The MNC band was harvested with a Pasteur pipette, and repeatedly washed with Hank's balanced salt solution.

\section{Measurement of inflammatory cytokines}

Plasma TNF- $\alpha$ and IL- 6 concentrations were measured with high-sensitivity ELISA kits (R\&D Systems, Minneapolis, MN, USA). Levels of plasma C-reactive protein were also measured with an ELISA kit (Alpha Diagnostic International, San Antonio, TX, USA). Plasma NEFA concentrations were measured using the Half-Micro colorimetric kit (Roche Diagnostics, Indianapolis, IN, USA).

\section{Measurement of reactive oxygen species}

Intracellular $\mathrm{H}_{2} \mathrm{O}_{2}$ was measured as described previously ${ }^{(17)}$. Briefly, $\mathrm{H}_{2} \mathrm{O}_{2}$ was measured using $2^{\prime}-7^{\prime}$-dichlorodihydrofluorescein diacetate. Mitochondrial peroxynitrite $\left(\mathrm{ONOO}^{-}\right)$was measured by dihydrorhodamine 123 (Invitrogen, Carlsbad, CA, USA). MNC were loaded with $10 \mu \mathrm{M}-2^{\prime}-7^{\prime}$-dichlorodihydrofluorescein diacetate and dihydrorhodamine 123 . Fluorescence intensity was measured with excitation at $488 \mathrm{~nm}$ and emission at $525 \mathrm{~nm}$ by a flow cytometer (FACSCalibur; Becton-Dickinson, Franklin Lakes, NJ, USA).

\section{Mitochondria fractionation}

The mitochondrial fraction and mitochondrial proteins were prepared as described earlier ${ }^{(18)}$. Freshly isolated MNC were suspended in lysis buffer (250 mm-sucrose, 0.1 mm-EDTA, 2 mM-HEPES, pH 7.4), homogenised, and then the mitochondria were separated from the cytosol by differential centrifugation. The isolated mitochondria were lysed in lysis buffer (Intron Biotechnology, Kyunggi, Korea).

\section{Measurement of malondialdehyde}

Malondialdehyde was measured using the thiobarbituric acid (TBA)-reactive substances assay kit (Cayman Chemical Company, Ann Arbor, MI, USA). Briefly, $100 \mu$ l of the cell lysates and $100 \mu \mathrm{l}$ of SDS solution were added in $4 \mathrm{ml}$ of colour reagent solution $(530 \mathrm{mg}$ TBA in $50 \mathrm{ml} \mathrm{TBA-acetic}$ acid solution and $50 \mathrm{ml} \mathrm{TBA}-\mathrm{NaOH})$. The samples were heated at $70^{\circ} \mathrm{C}$ for $60 \mathrm{~min}$ and then cooled at ice for $10 \mathrm{~min}$ before centrifuging at $1600 \mathrm{~g}$ for $10 \mathrm{~min}$. To measure the absorbance at $540 \mathrm{~nm}, 150 \mu \mathrm{l}$ of the supernatants were transferred to the microplate. 


\section{Western blot analysis}

Western blotting was performed with cytosolic and mitochondrial lysate. Monoclonal antibodies against NADH dehydrogenase [ubiquinone] $1 \beta$ sub-complex subunits 6 and 8 (NDUFB6 and NDUFB8), succinate dehydrogenase [ubiquinone] flavoprotein subunit $(70 \mathrm{kDa})$, cytochrome $b-c 1$ complex subunit 1 , cytochrome $b-c 1>$ complex subunit (Rieske Fe-S), cytochrome $c$ oxidase subunit 1 (COX1) (MitoSciences, Inc., Eugene, OR, USA), catalase (Calbiochem, Inc., La Jolla, CA, USA) and peroxiredoxins (Prx) 1, 2 and 3 (AbFrontier Company, Seoul, Korea) were used. Polyclonal antibodies against Mn-superoxide dismutase (SOD) and CuZn-SOD (Assay Designs, Inc., Ann Arbor, MI, USA), NADH dehydrogenase [ubiquinone] Fe-S protein 5 (Gene Tex, Inc., Irvine, CA, USA), thioredoxin $1, \alpha$-actinin, mitochondrial transcription factor A (TFAM), SOCS-3 and voltage-dependent anion-selective channel protein 1 (Santa Cruz Biotechnology, Santa Cruz, CA, USA) were used.

\section{Semi-quantitative $P C R$}

The transcript levels of NADH dehydrogenase 1 (ND1) of complex I, succinate dehydrogenase complex subunit $\mathrm{B}$ (SDHB) of complex II, COX1 of complex III, COX5B of complex IV and actin were measured using $\mathrm{SYBR}^{\circledR}$ Green PCR Master Mix (Applied Biosystems, Foster City, CA, USA) and the ABI PRISM ${ }^{\circledR} 7300$ real-time PCR system (Applied Biosystems), according to the manufacturer's instructions. The primer sequences designed by primer 3 and UCSC In-Silico PCR (http://genome.ucsc.edu) were as follows:

ND1 forward: 5'-TTC ATA GCC GAA TAC ACA AAC A-3'; ND1 reverse: ${ }^{\prime}$-GGG AGG TCA GAA GTA GGG TCT T- $3^{\prime}$; SDHB forward: 5'-TCA AAA ATC TAC CCT CTT CCA CA- $3^{\prime}$; SDHB reverse: $5^{\prime}$-TCC AGT TTC TCA CGC TCT TCT AT- $3^{\prime}$; COXI forward: 5'-GGT AAC GAC CAC ATC TAC AAC G-3'; COXI reverse: $5^{\prime}$-GGA GAG AGG GAG GTA AGA GTC A- $3^{\prime}$; COX5B forward: $5^{\prime}$-TGG TGT TCC CAC TGA TGA AG-3'; COX5B reverse: 5'-GAC GCT GGT ATT GTC CTC TT-3'; Actin forward: 5'-GCG ACG AGG CTC AGA GCA AG-3'; Actin reverse: 5'-CCG GAG GCG TAG AGG GAC AG-3'.

The thermal cycling conditions were as follows: $10 \mathrm{~min}$ at $95^{\circ} \mathrm{C}$ followed by fifty cycles of $95^{\circ} \mathrm{C}$ for $15 \mathrm{~s}$ and $60^{\circ} \mathrm{C}$ for $1 \mathrm{~min}$. Finally, the samples were held at $65^{\circ} \mathrm{C}$ for $5 \mathrm{~min}$ and melting curves were evaluated from 65 to $95^{\circ} \mathrm{C}$. Calculations were based on the 'Delta-Delta method' using the following equation ${ }^{(19)}$ :

$$
R(\text { ratio })=2^{-[\Delta \mathrm{CT} \text { sample- }-\Delta \mathrm{CT} \text { control }]} \text {. }
$$

The integrity of amplified DNA was confirmed by determination of the melting temperature. The data were expressed as fold change of the treatment groups in relation to the controls, and were normalised by levels of actin.

\section{Statistics}

All results were expressed as means and standard deviations. Error bars represent the means and standard deviations of three independent experiments. The difference between two mean values was analysed using Student's $t$ test. Differences were considered statistically significant when $P<0 \cdot 05$.

\section{Results}

All individuals showed normal glucose concentration in the oral glucose tolerance test on day 8 (data not shown). Also, glucose concentrations remained normal even after the intake of HFHC meals (Fig. 1(b)). However, fasting and glucose challenge insulin levels were highly elevated after the intake of HFHC meals for four consecutive days, compared with the intake of KDRI meals (Fig. 1(c) and (d)). Also, the expression level of SOCS-3 was increased after the intake of HFHC meals (Fig. 1(e)). Consistent with this, homeostasis model of assessment-insulin resistance (HOMA-IR), an index of insulin resistance, was increased after the intake of HFHC meals. The concentrations of HDL-cholesterol, LDL-cholesterol, total cholesterol and TAG were not significantly altered after the intake of HFHC meals. NEFA concentrations were significantly lowered after the intake of HFHC meals (Table 1). There were no changes in plasma TNF- $\alpha$, IL-6, or C-reactive protein concentrations following intake of either meal type (Table 1).

To determine the effect of excess energy following HFHC meals on ROS generation, ROS levels in MNC were measured by flow cytometric analysis. After an overnight fasting, ROS levels were measured at 0, 30, 60, 90 and $120 \mathrm{~min}$ following a single intake of the KDRI or HFHC meal at lunch. The $\mathrm{H}_{2} \mathrm{O}_{2}$ and $\mathrm{ONOO}^{-}$concentrations were significantly increased at 120 min after the single intake of the HFHC meal, compared with those after the single intake of the KDRI meal (Fig. 2(a) and (b)). To examine whether changes in $\mathrm{H}_{2} \mathrm{O}_{2}$ and $\mathrm{ONOO}^{-}$concentrations alter after the intake of the standardised diets, we performed the experiments with blood samples on days 1 and 7 for the standardised diets. As expected, the ROS levels did not change after the intake of KDRI meals for the standardised diet (Fig. 2(c) and (d)), indicating that values of the KDRI meal can be used as the control one. However, after the repeated intake of HFHC meals for $4 \mathrm{~d}$, levels of both $\mathrm{H}_{2} \mathrm{O}_{2}$ and $\mathrm{ONOO}^{-}$in $\mathrm{MNC}$ were decreased at days 9 and 11 by 60 (SD 4) and 42 (SD 6)\% (Fig. 2(e), $\mathbf{0}$ ), and 54 (SD 6) and 41 (SD 5)\% (Fig. 2(f), $\square)(P<0.05)$, respectively. After the intake of KDRI control meals, ROS levels did not change over this $4 \mathrm{~d}$ period (Fig. 2(e) and (f), $\square$ ). However, malondialdehyde concentration, an index of lipid peroxidation and oxidative stress, was increased after the intake of HFHC meals (Fig. 2(g)). The results showed that intracellular and mitochondrial ROS levels seemed to be similar in their patterns. However, we did not argue that absolute amounts of intracellular and mitochondrial ROS were the same because we measured both ROS levels with two different methods.

The mitochondrial antioxidant enzymes, Prx3 and Mn-SOD, were increased significantly after the intake of HFHC meals, compared with the control group (Fig. 3(a)). Protein levels of the cytosolic antioxidant enzymes, Prx1 and Prx2, were increased. However, levels of thioredoxin 1, catalase and CuZn-SOD were not changed following the intake of HFHC 
Table 1. Metabolic changes in normal lean individuals following the Dietary Reference Intake for Koreans (KDRI) or consuming high-fat, high-carbohydrate (HFHC) meals

(Mean values and standard deviations, $n 10$ )

\begin{tabular}{|c|c|c|c|c|c|c|c|c|}
\hline \multirow[b]{3}{*}{ Days } & \multicolumn{4}{|c|}{ KDRI } & \multicolumn{4}{|c|}{$\mathrm{HFHC}$} \\
\hline & \multicolumn{2}{|c|}{ Day 8} & \multicolumn{2}{|c|}{ Day 12} & \multicolumn{2}{|c|}{ Day 8} & \multicolumn{2}{|c|}{ Day 12} \\
\hline & Mean & SD & Mean & SD & Mean & SD & Mean & SD \\
\hline BMI $\left(\mathrm{kg} / \mathrm{m}^{2}\right)$ & $22 \cdot 78$ & 0.21 & $22 \cdot 81$ & 0.18 & $22 \cdot 73$ & 0.18 & 22.96 & 0.31 \\
\hline Weight (kg) & 70.56 & 2.23 & 70.66 & 2.37 & 72.02 & $2 \cdot 50$ & $72 \cdot 74$ & 2.01 \\
\hline Glucose $(\mathrm{mg} / \mathrm{l})$ & 887 & 71 & 87 & 86 & 830 & 55 & 838 & 72 \\
\hline Fasting insulin $(\mu \mathrm{U} / \mathrm{ml}) \dagger$ & 8.4 & 0.6 & 8.4 & 0.9 & 8.3 & 1.6 & $12 \cdot 6^{*}$ & $2 \cdot 8$ \\
\hline Post-challenge insulin $(\mu \mathrm{U} / \mathrm{ml}) \dagger$ & $102 \cdot 9$ & 23.5 & $100 \cdot 2$ & 18.5 & 99.3 & 27.9 & $181 \cdot 2^{*}$ & $50 \cdot 6$ \\
\hline HOMA-IR & 1.84 & 0.22 & 1.81 & 0.31 & $1 \cdot 71$ & 0.26 & $2 \cdot 61^{*}$ & 0.59 \\
\hline HDL-cholesterol (mg/l) & 530 & 62 & 516 & 55 & 567 & 93 & 601 & 101 \\
\hline LDL-cholesterol (mg/l) & 840 & 97 & 857 & 65 & 915 & 14 & 890 & 98 \\
\hline Cholesterol (mg/l) & 169 & 172 & 165 & 191 & 186 & 134 & 1723 & 161 \\
\hline TAG $(\mathrm{mg} / \mathrm{l})$ & 1150 & 195 & 1170 & 250 & 1210 & 244 & 1153 & 260 \\
\hline NEFA $(\mathrm{mmol} / \mathrm{l})$ & 3.5 & 0.4 & 3.5 & 0.3 & 3.8 & 0.5 & $2 \cdot 6^{\star}$ & 0.4 \\
\hline TNF- $\alpha(p g / m l)$ & $8 \cdot 1$ & 1.7 & $8 \cdot 2$ & 1.4 & $8 \cdot 4$ & $2 \cdot 2$ & $8 \cdot 34$ & $2 \cdot 2$ \\
\hline IL-6 (pg/ml) & 3.34 & 0.7 & 3.37 & 0.8 & 3.56 & 0.6 & 3.42 & 0.29 \\
\hline $\operatorname{CRP}(\mu \mathrm{g} / \mathrm{ml})$ & 1.34 & 0.2 & 1.35 & 0.3 & 1.43 & 0.3 & 1.41 & 0.3 \\
\hline
\end{tabular}

HOMA-IR, homeostasis model of assessment-insulin resistance; CRP, C-reactive protein.

*Mean values were significantly different from those of the KDRI group.

$\dagger 1 \mu \mathrm{U} / \mathrm{ml}=6.945 \mathrm{pmol} / \mathrm{l}$.

meals (Fig. 3(a)). TFAM is encoded by nuclear DNA and regulates mitochondrial DNA transcription, including the transcription of mitochondrial DNA genes encoding mETC complex proteins. The expression levels of this transcription enzyme were higher following the intake of HFHC meals (Fig. 3(b)). The mETC comprises four complexes (complex I-IV) and
ATP synthetase (complex V). Expression levels of NDUFB6, NDUFB8 and NADH dehydrogenase [ubiquinone] Fe-S protein 5 of the complex I subunit, $70 \mathrm{kDa}$ succinate dehydrogenase [ubiquinone] flavoprotein subunit of the complex II subunit, cytochrome $b-c 1$ complex subunit 1 and Rieske Fe-S of the complex III subunit, and COX1 of the complex IV subunit (a)

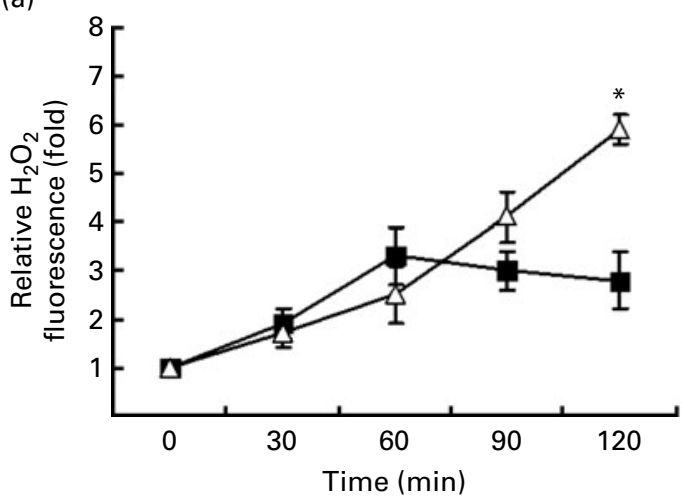

(b)

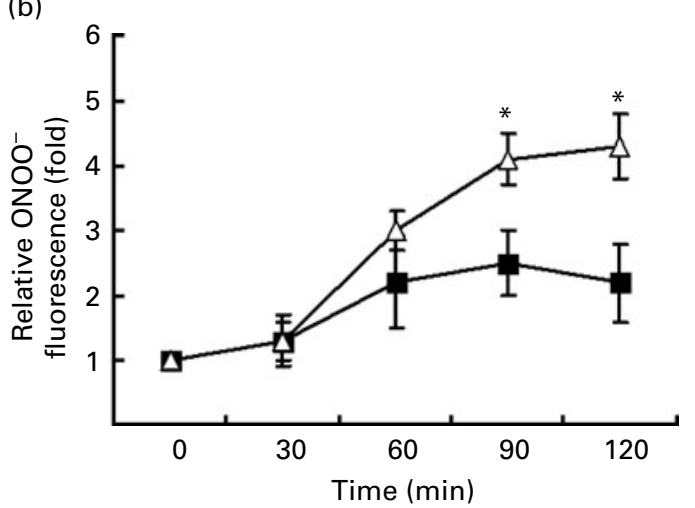

(f)

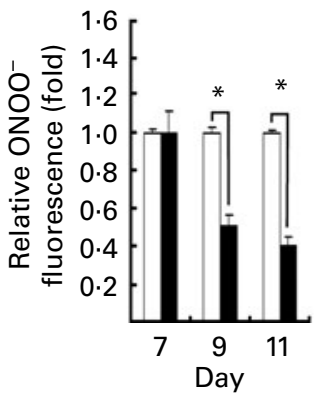

(g)

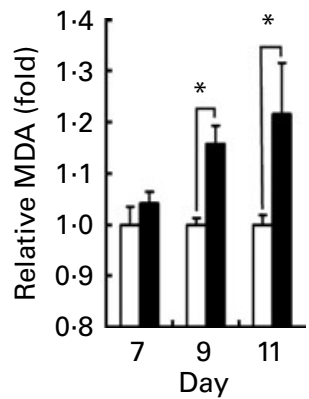

Fig. 2. Oxidative stress after the intake of high-fat, high-carbohydrate (HFHC) meals. Intracellular $\mathrm{H}_{2} \mathrm{O}_{2}\left(\mathrm{a}, \mathrm{c}\right.$ and e) and mitochondrial peroxynitrite $\left(\mathrm{ONOO}^{-}\right)$ levels (b, $d$ and $f$ ) in mononuclear cells were measured following a single intake of the HFHC (- - ) or Dietary Reference Intake for Koreans (KDRI, $-\mathbf{\square}-)$ meal ( $a$ and $b$ ), the standardised diet (days 1 and 7) (c and d) and the intake of the HFHC or KDRI meal (e and f). (g) Malondialdehyde (MDA) concentration was measured following the intake of the HFHC or KDRI meal. * Mean values were significantly different from those of the KDRI group $(P<0.05)$. 
(a)

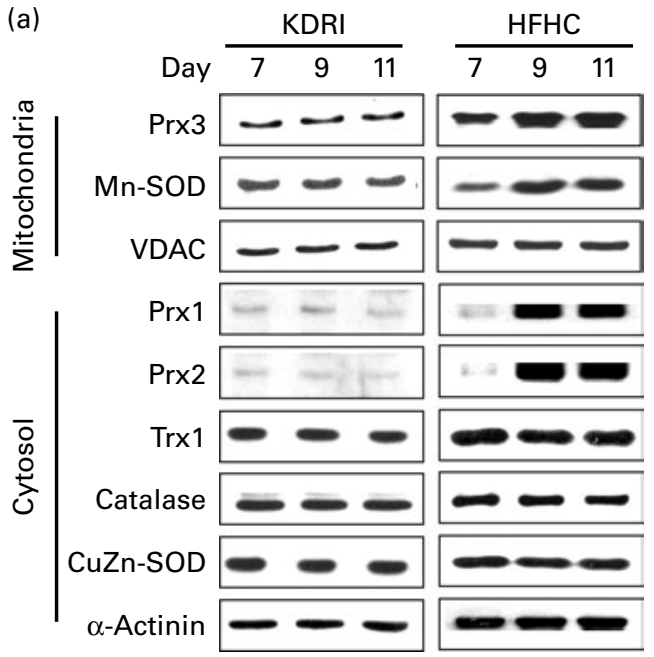

(b)

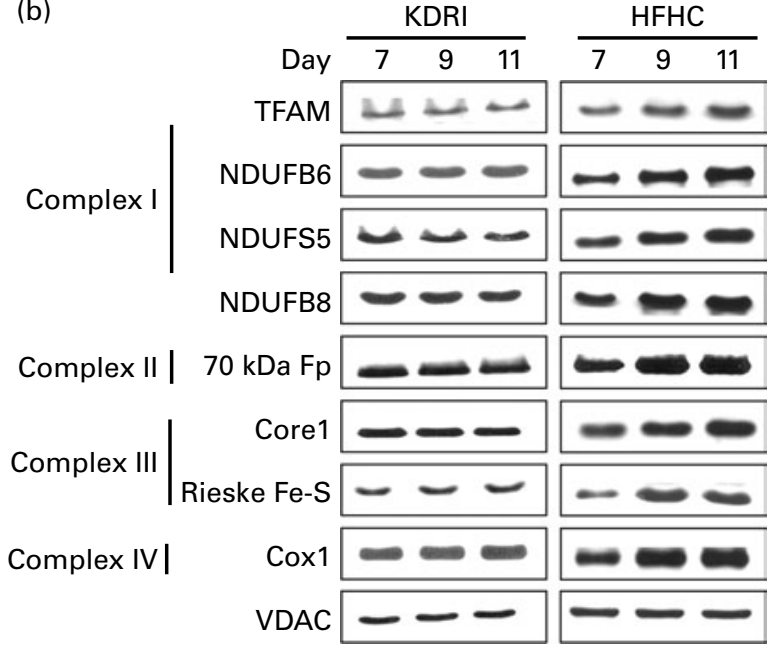

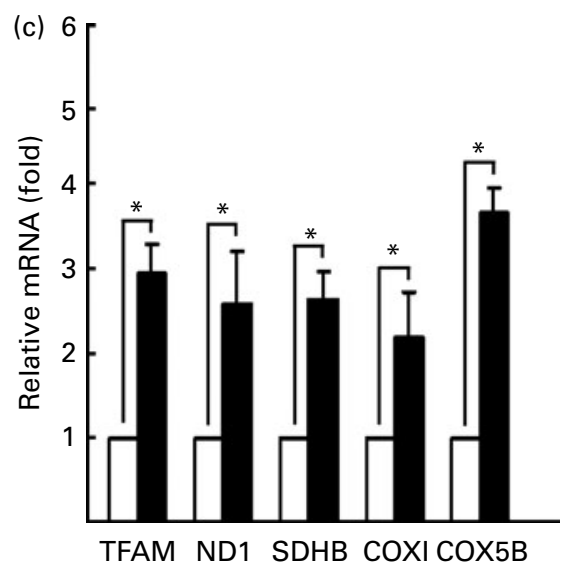

Fig. 3. Change in the expression levels of cytosolic and mitochondrial antioxidant enzymes, and mitochondrial electron transport chain ( $\mathrm{mETC}$ ) subunits. (a and b) Western blot analysis. Mitochondrial antioxidants (Mn-superoxide dismutase (SOD) and peroxiredoxin (Prx) 3), cytosolic antioxidants (Prx1, Prx2, thioredoxin 1 (Trx1), catalase and CuZn-SOD) (a), and mETC complex I, complex II, complex III, complex IV and mitochondrial transcription factor A (TFAM) (b) were measured at days 7, 9 and 11 following the intake of the high-fat, high-carbohydrate (HFHC) or Dietary Reference Intake for Koreans (KDRI) meal. $\alpha$-Actinin and voltage-dependent anion-selective channel protein (VDAC) were used as loading controls. (c) Real-time quantitative RT-PCR. The mETC complex I (NADH dehydrogenase 1 (ND1)), complex II (succinate dehydrogenase complex subunit B (SDHB)), complex III (cyclo-oxygenase subunit (COX)1), complex IV (COX5B) and TFAM were measured at days 7 and 11. * Mean values were significantly different from those of the KDRI group $(P<0.05)$. NDUFB, NADH dehydrogenase [ubiquinone] $1 \beta$ sub-complex subunit; NDUFS, NADH dehydrogenase [ubiquinone] Fe-S protein $5 ; 70 \mathrm{kDa} F \mathrm{p}$, succinate dehydrogenase [ubiquinone] flavoprotein subunit, Core1, cytochrome $b-c 1$ complex subunit 1; Rieske Fe-S, cytochrome $b-c 1$ complex subunit.

were also increased significantly after the intake of HFHC meals, compared with intake of KDRI meals (Fig. 3(b)). Voltage-dependent anion-selective channel protein was used to examine whether an equal amount of mitochondrial proteins was loaded for Western blot analysis. Real-time RT-PCR assays also showed that mRNA levels of mETC complex subunits were increased after the intake of HFHC meals (Fig. 3(c)).

\section{Discussion}

Our data confirm our hypotheses and provide several novel observations. We have shown that in association with an increase in ROS generation following intake of excess energy in the form of a HFHC meal, there is an increase in $\mathrm{ONOO}^{-}$generation as well ${ }^{(20)}$. In addition, following the intake of a sequence of such meals over $4 \mathrm{~d}$, several antioxidant enzymes as well as mitochondrial transcription factor,
TFAM, and mETC components were induced. Also, SOCS-3, the protein which interferes with insulin signal transduction, was induced in association with an increase in plasma insulin concentrations and HOMA-IR, the index of insulin resistance $^{(21)}$. In the present study, one group following excess energy intakes in the form of HFHC meals gained some weight compared with those of the other group. The results were not statistically significant. However, if individuals ingest HFHC meals continuously, we may get statistically significant results.

The observation on the acute induction of $\mathrm{ONOO}^{-}$by the single intake of the HFHC meal is novel similar to that on the induction of antioxidant enzymes after repeated intake of HFHC meals. The induced antioxidant enzymes were both from the mitochondria and the cytosol. The major mitochondrial antioxidant enzymes induced were Prx3 and MnSOD. The increased expression of these genes would be 
protective in the context of the marked increase in ROS generation by the mitochondria following the intake of HFHC meals. This is important since mitochondria are the main sources of intracellular ROS and, therefore, are vulnerable to oxidative stress and injury. The induction of cytosolic Prx1 and Prx 2 would protect cytosolic contents from oxidative damage. However, we were surprised to find no change in catalase that converts $\mathrm{H}_{2} \mathrm{O}_{2}$ to water. In addition, there was no change in thioredoxin 1 and CuZn-SOD expression.

Although the induction of these antioxidant enzymes was associated with a reduction of $\mathrm{H}_{2} \mathrm{O}_{2}$ and $\mathrm{ONOO}^{-}$after the repeated intake of excess energy in the form of HFHC meals, there was a persistent increase in the cellular content of malondialdehyde. This is indicative of increased lipid peroxidation and oxidative stress. Clearly, the compensatory mechanisms triggered by the meals are not sufficient to prevent or reverse the oxidative stress induced by the meals. In this context, the recent data showing the ability of orange juice to suppress ROS generation, oxidative stress and inflammation induced by the single intake of a HFHC meal is of interest $^{(22)}$. Whether this beneficial effect of orange juice is mediated by the induction of the antioxidant enzymes, as shown in the present study, needs to be tested.

Our data also show for the first time that excess energy in the form of HFHC meals induces the major mitochondrial transcription factor, TFAM, that regulates the transcription of the mitochondrial genome, including the genes involved in the mETC. There was an induction of NDUFB6, NDUFB8, NDUFFS5 from ETC complex I, $70 \mathrm{kDa}$ succinate dehydrogenase [ubiquinone] flavoprotein subunit from complex II, cytochrome $b-c 1$ complex subunit 1 and Rieske Fe-S from complex III and COX1 from complex IV at the protein levels. In addition, there was an increase in ND1, COX5B and SDHB at mRNA levels. This is relevant to the transcription of the mitochondrial metabolic function that intrinsically involves the generation of $\mathrm{O}_{2}^{-}$radical $^{(23)}$. It is of interest that the increased metabolic activity induced by HFHC meals associated with the increase in ROS generation is synchronised with an increase in the induction of the antioxidant enzymes mentioned above. Since the $\beta$-cell in the pancreatic islet is not endowed with adequate antioxidant mechanisms, it is relatively vulnerable to oxidative stress and injury ${ }^{(24,25)}$. It is of interest that TFAM deletion leads to diabetes in mice ${ }^{(26)}$.

The induction of SOCS-3 by excess energy intakes in the form of HFHC meals demonstrates that such meals induce molecules that interfere with insulin signal transduction and contribute to the pathogenesis of insulin resistance. Indeed, the intake of these meals led to an increase in insulin concentrations and HOMA-IR. Thus, the combination of oxidative stress and inflammation leading to an increase in SOCS-3 expression and HOMA-IR provides a framework within which we can begin to understand the mechanisms underlying the pathogenesis of diet-related insulin resistance.

Our data are somewhat limited because we did not have any data on dietary and exercise habits of individuals before participation in the present study. Actually, dietary and exercise habits affect ROS generation and inflammation. However, this fact does not affect the validity of our data because both groups stayed in a camp to prohibit unnecessary action and ingested the standardised diet for $7 \mathrm{~d}$ to nullify any effects caused by dietary and exercise habits. In the present study, excess energy was mainly due to increased fat ingestion. At this time, we do not know whether excess energy or increased fat directly caused the results. To solve the question, more studies need to be conducted in the future.

In conclusion, we have demonstrated for the first time that while sequential excess energy intakes in the form of HFHC meals induce oxidative stress, they also induce mitochondrial and cytosolic antioxidant enzymes and the major mitochondrial transcription factor, TFAM. This is associated with the induction of the proteins involved in mETC. Nevertheless, there is an increase in intracytosolic lipid peroxidation and the induction of SOCS-3, which interferes with insulin signal transduction. This is associated with an increase in HOMA-IR, consistent with the induction of insulin resistance. This simple model of insulin resistance caused by a rapid induction of excess energy in the form of a HFHC meal will be used for further investigation and to arouse people's attention to the detrimental effects of the HFHC meal.

\section{Acknowledgements}

The present study was supported by a Korea Science and Engineering Foundation grant funded by the Korean government (MEST) (no. 20090091346) to S. S. K. The authors' responsibilities were as follows: S. L. and S. S. K. designed the research; S. L. performed the research; S. L., H. W., Ye. K., M. J., K. R. J., Yo. K., P. D., J. H. and S. S. K. analysed the data; and S. L., P. D. and S. S. K. wrote the manuscript. All authors read and approved the final manuscript. There are no conflicts of interest.

\section{References}

1. Slim RM, Toborek M, Watkins BA, et al. (1996) Susceptibility to hepatic oxidative stress in rabbits fed different animal and plant fats. J Am Coll Nutr 15, 289-294.

2. Mohanty P, Hamouda W, Garg R, et al. (2000) Glucose challenge stimulates reactive oxygen species (ROS) generation by leucocytes. J Clin Endocrinol Metab 85, 2970-2973.

3. Dandona P, Ghanim H, Chaudhuri A, et al. (2010) Macronutrient intake induces oxidative and inflammatory stress: potential relevance to atherosclerosis and insulin resistance. Exp Mol Med 42, 245-253.

4. Ghanim H, Abuaysheh S, Sia CL, et al. (2009) Increase in plasma endotoxin concentrations and the expression of toll like receptors and suppressor of cytokine signaling-3 in mononuclear cells following a high fat high carbohydrate meal: implications for insulin resistance. Diabetes Care 32 , 2281-2287.

5. Lazar MA (2005) How obesity causes diabetes: not a tall tale. Science 307, 373-375.

6. Pereira MA, Kartashov Al, Ebbeling CB, et al. (2005) Fastfood habits, weight gain, and insulin resistance (the CARDIA study): 15-year prospective analysis. Lancet $\mathbf{3 6 5}$, 36-42.

7. Marshall JA, Hamman RF \& Baxter J (1991) High-fat, lowcarbohydrate diet and the etiology of non-insulin-dependent 
diabetes mellitus: the San Luis Valley Diabetes Study. Am J Epidemiol 134, 590-603.

8. Kim JK, Gimeno RE, Higashimori T, et al. (2004) Inactivation of fatty acid transport protein 1 prevents fat-induced insulin resistance in skeletal muscle. J Clin Invest 113, 756-763.

9. Cornier MA, Bergman BC \& Bessesen DH (2006) The effects of short term overfeeding on insulin action in lean and reduced obese individuals. Metabolism 55, 1207-1214.

10. Brons C, Jensen CB, Storgaard H, et al. (2009) Impact of short-term high-fat feeding on glucose and insulin metabolism in young healthy men. $J$ Physiol 587, 2387-2397.

11. Schrauwen P \& Hesselink MKC (2004) Oxidative capacity, lipotoxicity and mitochondrial damage in type 2 diabetes. Diabetes 53, 1412-1417.

12. Wilde JD, Mohren R, Berg SVD, et al. (2008) Short-term high fat-feeding results in morphological and metabolic adaptations in the skeletal muscle of C57BL/6J mice. Physiol Genomics 32, 360-369.

13. Lee B, Srinivasan M, Aalinkeel R, et al. (2001) Mitochondrialencoded gene regulation in rat pancreatic islets. Metabolism 50, 200-206.

14. Kim L, Deng H, Fukushima A, et al. (2009) Reactive oxygen species enhance insulin sensitivity. Cell Metabolism 10, 260-272.

15. Anderson EJ, Lustig ME, Boyle KE, et al. (2009) Mitochondrial $\mathrm{H}_{2} \mathrm{O}_{2}$ emission and cellular redox state link excess fat intake to insulin resistance in both rodents and humans. J Clin Invest 119, 573-581.

16. The Korean Nutrition Society (2010) Dietary reference intakes for Koreans. http://image.campushomepage.com/ users/knsweb/ssugi513/DRIs/2010KDRIs_open_final.pdf

17. Ding Y, Choi KJ, Kim JH, et al. (2008) Endogenous hydrogen peroxide regulates glutathione redox via nuclear factor erythroid 2-related factor 2 downstream of phosphatidylinositol 3-kinase during muscle differentiation. Am J Pathol 172, 1529-1541.

18. Choi KJ, Piao YJ, Lim MJ, et al. (2007) Overexpressed cyclophilin A in cancer cells renders resistance to hypoxia- and cisplatin-induced cell death. Cancer Res 67, 3654-3662.

19. Livak KJ \& Schmittgen TD (2001) Analysis of relative gene expression data using real-time quantitative PCR and the 2(-Delta Delta C(T)) method. Methods 25, 402-408.

20. Patel C, Ghanim H, Ravishankar S, et al. (2007) Prolonged reactive oxygen species generation and nuclear factor- $\mathrm{\kappa B}$ activation after a high-fat, high-carbohydrate meal in the obese. J Clin Endocrinol Metab 92, 4476-4479.

21. Emanuelli B, Peraldi P, Filloux C, et al. (2000) SOCS-3 is an insulin-induced negative regulator of insulin signaling. J Biol Chem 275, 15985-15991.

22. Ghanim G, Sia CL, Upadhyay M, et al. (2010) Orange juice neutralizes the proinflammatory effect of a high-fat, highcarbohydrate meal and prevents endotoxin increase and toll-like receptor expression. Am J Clin Nutr 91, 940-949.

23. Huang G, Chen Y, Lu H, et al. (2007) Coupling mitochondrial respiratory chain to cell death: an essential role of mitochondrial complex $I$ in the interferon-beta and retinoic acidinduced cancer cell death. Cell Death Differ 14, 327-337.

24. Grankvist K, Marklund SL \& Taljedal IB (1981) CuZnsuperoxide dismutase, Mn-superoxide dismutase, catalase and glutathione peroxidase in pancreatic islets and other tissues in the mouse. Biochem J 199, 393-398.

25. Tiedge M, Lortz S, Drinkgern J, et al. (1997) Relation between anti-oxidant enzyme gene expression and antioxidative defense status of insulin-producing cells. Diabetes 46, 1733-1742.

26. Silva JP, Köhler M, Graff C, et al. (2000) Impaired insulin secretion and $\beta$-cell loss in tissue-specific knockout mice with mitochondrial diabetes. Nat Genet 26, 336-340. 